\title{
Study of Contact Resistance of High-mobility Organic Transistors through Comparisons
}

Takafumi Matsumoto ${ }^{1,2}$, Wei Ou-Yang ${ }^{1, a}$, Kenjiro Miyake ${ }^{1}$, Takafumi Uemura $^{1}$, and Jun Takeya ${ }^{1, b}$

${ }^{1}$ Graduate School of Frontier Sciences, The University of Tokyo, 5-1-5, Kashiwanoha, Kashiwa, Chiba 277-8561, Japan

${ }^{2}$ Central Research Center, Toppan Forms Co. Ltd., 1-2-6 Owada-Cho, Hachioji, Tokyo 192-0045, Japan

\begin{abstract}
Realization of high-frequency low-cost organic electronics requires high-mobility organic field-effect transistors (OFETs) with short channels, where influence of contact resistance becomes more serious than either lower mobility or longer channel devices. To reduce the contact resistance, we systematically and quantitatively investigate the influence of the lowest unoccupied molecular orbital (LUMO) level of an electron acceptor layer, the active layer thickness, and the side chain of active layer itself on contact resistance of top-contact high-mobility OFETs through a series of comparative analysis. We find that the acceptor of $1,3,4,5,7,8$ - hexafluoro tetracyano naphtha quinodimethane $\left(F_{6}\right.$ TNAP) with a deeper LUMO level is efficient for carrier injection and that the bulk resistance plays an important role in such devices. By optimizing the parameters, we get the lowest contact resistance of only $110 \Omega \cdot \mathrm{cm}$, and thus recorded effective mobility of $8.0 \mathrm{~cm}^{2} / \mathrm{Vs}$ is attained for polycrystalline thin film transistors and still kept as high as $6 \mathrm{~cm}^{2} / \mathrm{Vs}$ at shorter channel lengths.
\end{abstract}

Keywords: contact resistance; organic field-effect transistor; DNTT; bulk resistance; electron acceptor; alkyl side chain

\footnotetext{
a w-ouyang@organicel.k.u-tokyo.ac.jp

b takeya@k.u-tokyo.ac.jp 


\section{Introduction}

Organic field-effect transistors (OFETs) acting as core components of the newly booming flexible organic electronics are receiving more and more attentions for potential foldable products in coming smart society fabricated with low cost on large-area rollable substrates $[1,2]$. Such products as planar displays and radio frequency indentification (RFID) require the electronics to respond in a short time [3]. In other words, high operation speed is a prerequisite for the switching OFET components. As one solution, many efforts have been made to improve carrier mobility to $10 \mathrm{~cm}^{2} / \mathrm{Vs}[4-8]$, over one order in magnitude higher than currently commercialized hydrogenated amorphous silicon (a-Si:H) based electronics. Dinaphtho[2,3-b:2',3'-f]thieno[3,2-b]thiophene (DNTT) as a newly developed $p$-type small-molecule organic semiconductor has a very high field-effect mobility of 2.9 $\mathrm{cm}^{2} / \mathrm{Vs}[9]$ in evaporated thin film phase and recently it has been shown that its alkyl derivative of 2,9-didecyl-dinaphtho[2,3-b:2',3'-f]thieno[3,2-b]thiophene $\left(\mathrm{C}_{10}\right.$-DNTT) in the phase of single-crystalline possesses a much higher intrinsic mobility over 10 $\mathrm{cm}^{2} / \mathrm{Vs}[7,8]$. The great increase of carrier mobility was assigned to better molecular ordering in the presence of long alkyl chains [10]. However, such alkyl chain effect is somewhat complex and sometimes ambiguous discussions have been made [11-13]. For instance, Kaneto et al claimed that the insulating side alkyl chains prohibit carrier migration [11]. Additionally, information about influence of the alkyl chain on contact and/or channel resistance is unknown, which is important for understanding effect of the side chain and thus crucial for the optimization of molecular design.

As another solution, there is an increasing tendency to employ short channel devices

since the operational frequency is proportional to $L^{-2}$ (L: channel length) [14]. However, 
the short channel devices usually meet the problem of carrier injection due to high contact resistance, which greatly suppresses the effective carrier mobility and influences the saturation of output current [14-16]. This is the reason that even if a semiconductor is known to possess a much larger intrinsic mobility, the commonly reported effective mobility for such short channel devices is below $1 \mathrm{~cm}^{2} / \mathrm{Vs}$ and thus high cutoff frequency in the range of $10 \mathrm{MHz}$ is rarely reported with some exceptions [15,17-19 ]. For example, the DNTT has been demonstrated to have a high intrinsic mobility of $\sim 3 \mathrm{~cm}^{2} \mathrm{Ns}$ in normal channel OFETs $[9,15]$, while the effective mobility decreases to $0.2 \sim 0.3 \mathrm{~cm}^{2} / \mathrm{Vs}$ for short channel devices $[15,19]$ due to the increased role of contact resistance. The contact resistance is conventionally evaluated with the transmission line method (TLM) from linear regime [20], and recently a modified TLM was proposed to overcome the difficulties of TLM and more accurate results were reported [21]. So far, it has been reported that many origins are responsible for the generation of contact resistance such as source electrode - organic semiconductor interfacial energy states [22], the presence of dipole layers at the interface [23], and bulk resistance of the organic active layer itself [24]. To reduce the contact resistance, many methods have been reported. For instance, by inserting a doping/buffer layer such as 2,3,5,6-tetrafluoro-7,7,8,8,-tetracyanoquinodimethane ( $F_{4}$ TCNQ) [24], iron (III) chloride $\left(\mathrm{FeCl}_{3}\right)$ [25], and others [26,27], the contact resistance has been proven decreased. In addition, though it is commonly accepted that only the first few monolayers of active molecules play paramount role in carrier transport [28], the active layer thickness, which has been demonstrated as an important parameter to get optimized device's performance [29,30], has received less attention, especially its influence on contact resistance for high-mobility devices. 
In this study, we comprehensively investigate a few factors influencing contact resistance of high-mobility OFETs including different electron acceptor layers, active layer thickness, and the saturated alkyl chain through a series of comparison studies. Results show the electron acceptor with a deeper lowest unoccupied molecular orbital (LUMO) level is more efficient for carrier injection, and bulk resistance dominates contact resistance in the devices with a thick active layer and thus a thinner active layer is necessary for reducing the contact resistance. In addition, great impact of the side alkyl chains on channel resistance rather than contact resistance is observed.

\section{Experiments and Methods}

We fabricated top-contact bottom-gate (TC/BG) OFETs of DNTT and its alkyl derivative of $\mathrm{C}_{10}$-DNTT since it has been demonstrated the derivative with two decyl chains has the highest mobility [10]. The schematic illustration of device structure is shown in the inset of Fig. 1(a). Highly doped silicon wafers with a thermally grown $\mathrm{SiO}_{2}$ layer of $200 \mathrm{~nm}$ was used as substrates. A substrate was cleaned first through acetone and isoproponol wash under ultra sonication, and then with a $\mathrm{N}_{2}$ blow and a subsequent drying process on a hotplate. After cleaning, it was transferred to a UV/ozone chamber for $30 \mathrm{~min}$ to achieve an atomic-level clean and hydrophilic surface. Subsequently, decyltricholorosilane self-assembled monolayers (DTS-SAMs) were coated to passivate the silicon dioxide surface immediately and meanwhile to change the surface to hydrophobic for organic semiconductor layer deposition. The SAM process was done in an oven kept at $120^{\circ} \mathrm{C}$ for $3 \mathrm{~h}$. For the organic active layer, DNTT or $\mathrm{C}_{10}$-DNTT thin films were formed by thermally evaporating their powders to the upward mounted substrates which were kept at an elevated temperature of $80^{\circ} \mathrm{C}$. The thermal evaporation was carried out in high vacuum at a deposition rate of $0.5 \AA / s$ 
regulated with a quartz crystal microbalance (QCM). To investigate the interfacial energy level alignment, we chose two kinds of electron acceptors, namely commonly used $\mathrm{F}_{4} \mathrm{TCNQ}$ and newly developed $1,3,4,5,7,8$ - hexafluoro tetracyano naphtha quinodimethane $\left(\mathrm{F}_{6}\right.$ TNAP), who have a deep LUMO level of -5.35 and $-5.37 \mathrm{eV}$, respectively [31]. The acceptor layer and top gold electrodes were thermally evaporated in vacuum through a metal mask with a thickness of 1 and $30 \mathrm{~nm}$, respectively. Designed devices have a channel width (W) of $500 \mu \mathrm{m}$ and a channel length varying from 20 to $1000 \mu \mathrm{m}$. The output and transfer characteristics were obtained in dark under ambient atmosphere using a program for current-voltage $(I-V)$ measurement equipped in the Keithley 4200 semiconductor characterization system. The organic active layer thickness was checked by atomic-force microscopy (AFM) in a dynamic force mode (DFM) (SPI 3800N NanoNavi, SII NanoTechnology Inc.).

For contact resistance extraction, instead of conventional TLM method, we employ the modified TLM (M-TLM) method which has shown better accuracy [21]. The total drain-source resistance $\left(R_{t o t}\right)$ is given as a sum of the contact resistance $\left(R_{c}\right)$ and the channel resistance $\left(R_{c h}\right)$ and it is expressed as

$$
R_{\text {tot }} W / L=R_{c} W \frac{1}{L}+\frac{1}{C_{i}\left(V_{G}-V_{t h}\right) \mu_{l i n}}
$$

in linear regime $\left(\left|V_{D}\right|<<\left|V_{G}-V_{t h}\right|\right)$. In the equation, $V_{D}, V_{G}$ and $V_{t h}$ are applied drain voltage, gate voltage and threshold voltage, respectively; $C_{i}$ is the areal capacitance of gate insulator, and $\mu_{\text {lin }}$ is the effective mobility estimated from the linear regime. The contact resistance thus can be extracted as the vertical intercept by fitting the channel length dependent total resistance. 


\section{Results and discussion}

Figure 1 shows typical $I-V$ results of an OFET with $C_{10}$-DNTT active layer of $15 \mathrm{~nm}$. The carrier mobility in linear regime and saturated regime $\left(\mu_{\text {sat }}\right)$ are estimated by linear fitting in Fig. 1(a) and (b) using the following relations:

$$
\begin{array}{cc}
I_{D}=\frac{W \mu_{l i n} C_{i}}{L}\left(V_{G}-V_{t h}-\frac{1}{2} V_{D}\right) V_{D}, & \left(\left|V_{D}\right|<<\left|V_{G}-V_{t h}\right|\right) \\
\sqrt{I_{D}}=\sqrt{\frac{W \mu_{s a t} C_{i}}{2 L}}\left(V_{G}-V_{t h}\right), & \left(\left|V_{D}\right|>>\left|V_{G}-V_{t h}\right|\right)
\end{array}
$$

where $I_{D}$ is drain-source current. The obtained mobilities in linear and saturated regimes for the OFET device with an F $_{6}$ TNAP electron acceptor layer are about 7.0 and $8.0 \mathrm{~cm}^{2} / \mathrm{Vs}$, respectively. Though high mobility over $10 \mathrm{~cm}^{2} / \mathrm{Vs}$ has been reported for the $\mathrm{C}_{10}$-DNTT OFETs on different dielectrics, such high mobility was achieved only in the phase of single crystalline $[7,8]$. Hence, as far as we know, these values are highest achieved for evaporated multicrystalline thin film. It is worthy to note that while the reported highest mobility for the material in linear regime was about $4 \mathrm{~cm}^{2} / \mathrm{Vs}$ $[8,10]$, current study presents a much higher value, quite close to that got from saturated regime. The high mobility in linear region indicates the injection barrier has been greatly depressed in the operation mode of low drain voltage [8], which reflects the success of employing the electron acceptor layer of $F_{6}$ TNAP. The threshold voltage $\left(V_{t h}\right)$ obtained from saturated regime with Eq. (3) as shown in Fig. 1(b) were $-10 \mathrm{~V}$, which is almost the same as previously reported [10]. In addition, the on/off current ratio and subthreshold swing [32] are $10^{6}$ and around $1.0 \mathrm{~V} /$ decade, respectively. The output current curves shown in Fig. 1(c) present ideal saturation tendency and corresponds well with the estimated threshold voltage since almost no current flows until gate voltage goes up to $-10 \mathrm{~V}$. Here, for a clear comparison in the influence of different electron acceptor layers we also plotted the transfer lines of an 
$\mathrm{F}_{4}$ TCNQ inserted OFET in linear and saturated regimes in Fig. 1(a) and (b), respectively. The drain-source current is obviously depressed which is mostly by reason of threshold voltage shift rather than the change of effective mobility. This shift of threshold voltage is due to the change of internal electric field by replacing the electron acceptor layer which results in variation of contact resistance $[32,33]$.

To further investigate the effect of inserted electron acceptor layer, contact resistance analysis was made by employing the method of modified TLM in linear regime. Typical linear relations of total resistance with respect to reciprocal of channel length are demonstrated for a $\mathrm{C}_{10}$-DNTT OFET in Fig. 2. The channel length dependent dots (total contact resistance) for a certain gate voltage can be well linearly fitted and thus the contact resistances can be obtained with the method described in Eq. (1).

Figure 3(a) shows estimated contact resistance for thin-film $\mathrm{C}_{10}$-DNTT (15 nm) OFETs with an electron acceptor layer of $\mathrm{F}_{4} \mathrm{TCNQ}$ or $\mathrm{F}_{6} \mathrm{TNAP}$. Clearly, prominent decrease of contact resistance almost by half is observed for devices using $F_{6}$ TNAP as the acceptor layer compared with those incorporating an $\mathrm{F}_{4} \mathrm{TCNQ}$ layer. The smaller contact resistance can be explained by the reduced injection barrier between the acceptor layer (LUMO level) and organic semiconductor layer (highest occupied molecular orbital, HOMO: $5.38 \mathrm{eV}$ [11]) due to more deep LUMO level of the F $_{6}$ TNAP (5.37 eV) compared with $\mathrm{F}_{4} \mathrm{TCNQ}(5.35 \mathrm{eV})$. This proves the capability of reducing contact resistance by employing an optimally designed acceptor layer for an OFET. In addition, the obtained contact resistances for both devices are very small since best value is approximately $1 \mathrm{k} \Omega \cdot \mathrm{cm}$ such as for gold top-contact pentacene OFETs [22]. Actually, as far as we know, present contact resistance using F $_{6}$ TNAP as an electron 
acceptor layer is the lowest among reported values $[15,22,26]$. These small contact resistances result in almost smooth carrier injection and thus the high carrier mobility over $7 \mathrm{~cm}^{2} / \mathrm{Vs}$, approaching to its intrinsic value [7,8] can be achieved, as shown in Fig. 3(b). Compared with the case of $\mathrm{F}_{4} \mathrm{TCNQ}$, the slight enhanced mobility in the case of $F_{6}$ TNAP is due to smoother carrier injection as indicated by the lower contact resistance. In addition, as channel length decreases, the effective mobility is just slightly suppressed. This so-called short channel effect due to increased importance of contact resitance was found very serious as we introduced for DNTT case, and for $\mathrm{C}_{10}$-DNTT OFETs it has also been shown the effective mobility decreases by half from $7.6 \mathrm{~cm}^{2} / \mathrm{Vs}$ for a channel length of $190 \mu \mathrm{m}$ to just $3.7 \mathrm{~cm}^{2} / \mathrm{Vs}$ for a channel length of 40 $\mu \mathrm{m}$ [10], while in present case the mobility can still keep as high as $6 \mathrm{~cm}^{2} / \mathrm{Vs}$ thanks to the employment of strong acceptor layer and optimized layer thickness.

Since the above discussions neglect the contribution of bulk resistance of thin active layer itself which does contribute to the contact resistance, we compare the contact resistance of $\mathrm{C}_{10}$-DNTT OFETs with different active layer thicknesses. The OFETs with thicker active layer present much higher contact resistance as shown in Fig. 3(c). The additional contact resistance generated from the increased layer thickness of the active layer contributes to two third of the contact resistance indicating the bulk resistance plays a main role in contact resistance for such devices and thus for high-mobility OFET devices the active layer thickness is very important, especially in the case of smooth injection. To make its effect more clearly, we propose a simple equivalent circuit model where the contact resistance is composed of interfacial resistance $\left(R_{\text {int }}\right)$ and bulk resistance $\left(R_{b}\right)$ under source and drain electrodes, as illustrated in the inset of Fig. 3(c). In other words, there is a relation of $R_{c}=R_{\text {int }}+R_{b}$, 
and $R_{b}$ is a dominant component in the smooth injection high-mobility devices. In this sense, to fabricate a high-mobility device the active layer thickness must be optimized to make a compromise between carrier injection $\left(R_{c}\right)$ and carrier transport (carrier density).

Furthermore, though alkylized $\mathrm{C}_{10}$-DNTT OFETs have been proved to have larger mobility $[7,8,10]$, the alkyl chain effect has not been fully understood, especially on contact resistance or channel resistance. For instance, Cho et al claimed that the number of orientated active layers for effective carrier transport was also important and thus they observed higher mobility of poly(3-alkylthiophene) (P3AT) OFETs with shorter alkyl chain though the material itself had less ordered phase [34]. Figure 4(a) shows the results of contact resistance for OFETs with a DNTT layer or a C10-DNTT layer. They have almost the same contact resistance, which implies the alkyl chain does not influence contact resistance so much maybe due to the saturated alkyl groups have little influence on HOMO level [35]. To understand the relation of the greatly improved mobility (see Supporting Materials, Fig. S1(a)) in the viewpoint of resistance, we plot the channel length dependent contact resistance ratio in Fig. 4(b). The higher partial of contact resistance over total resistance for alkylized DNTT OFETs indicates there is smaller channel resistance, namely larger channel conductance, which agrees with the observed higher mobility. The agreement clearly shows the alkyl side chains have no impact on carrier density but have strong effect on effective mobility (see Supporting Materials, Fig. S1(a) and (b)). The observed enhancement of carrier mobility (or channel conductance) in the presence of insulating alkyl chain can be due to the suppression of Fröhlich polaronic coupling effect [8] and enhanced $\pi-\pi$ orbital coupling in the reason of better molecular ordering 
as revealed by $\mathrm{x}$-ray diffraction (XRD) study [10].

\section{Conclusions}

In this study we intensively investigated contact resistance of high-mobiltiy DNTT OFET devices under influence of active material itself, thickness of the active material and an electron acceptor interlayer with the modified transmission line method. Results show the OFETs with thinner active layer have smaller contact resistance and incorporation of an acceptor like F $_{6}$ TNAP with a deeper LUMO level is more efficient in contact resistance reduction. In addition, the side alkyl chain is proved to affect channel resistance without changing carrier density rather than contact resistance. These findings result in the success of fabricating devices with high effective mobility up to $8 \mathrm{~cm}^{2} / \mathrm{Vs}$ for polycrystalline film and not sacrificed heavily as previously reported in short channel devices due to the lowest contact resistance of just $110 \Omega \cdot \mathrm{cm}$.

\section{Acknowledgement}

We thank Nippon Kayaku Co. Ltd. for supplying $C_{10}$-DNTT. We acknowledge the New Energy and Industrial Technology Developing Organization (NEDO), the Japan Science and Technology Agency (JST), and the Ministry of Education, Culture, Sports, Science, and Technology (MEXT), Japan for financial support. 


\section{Reference}

[1] Z. Bao, J. Locklin, Organic Field-Effect Transistors, CRC Press, New York, 2007.

[2] J. Takeya, Printing Technologies for Organic Devices, CMC Press, Tokyo, 2012.

[3] W. Fix, A. Ullmann, J. Ficker, W. Clemens, Appl. Phys. Lett. 81 (2002) 1735.

[4] J. Takeya, M. Yamagishi, Y. Tominari, R. Hirahara, Y. Nakazawa, T. Nishikawa, T. Kawase, T. Shimoda, Appl. Phys. Lett. 90 (2007) 102120

[5] H. Minemawari, T. Yamada, H. Matsui, J. Tsutsumi, S. Haas, R. Chiba, R. Kumai, T. Hasegawa, Nature 475 (2011) 364

[6] C. Liu, T. Minari, X. Lu, A. Kumatani, K. Takimiya, K. Tsukagoshi, Adv. Mater. 23 (2011) 523.

[7] K. Nakayama, Y. Hirose, J. Soeda, M. Yoshizumi, T. Uemura, M. Uno, W. Li, M. J. Kang, M. Yamagishi, Y. Okada, E. Miyazaki, Y. Nakazawa, A. Nakao, K. Takimiya, and J. Takeya, Adv. Mater. 23 (2011) 1626.

[8] W. Ou-Yang, T. Uemura, K. Miyake, S. Onish, T. Kato, M. Katayama, M. Kang, K. Takimiya, M. Ikeda, H. Kuwabara, M. Hamada, J. Takeya, Appl. Phys. Lett. 101 (2012) 223304

[9] T. Yamamoto and K. Takimiya, J. Am. Chem. Soc. 129 (2007) 2224.

[10] M. J. Kang, I. Doi, H. Mori, E. Miyazaki, K. Takimiya, M. Ikeda, and H. Kuwabara, Adv. Mater. 23, (2011) 1222.

[11] K. Kaneto, W. Y. Lim, W. Takashima, T. Endo, M. Rikukawa, Jpn. J. Appl. Phys., 39 (2000) L872

[12] A. Babel, S. A. Jenehe, Synth. Met. 148 (2005) 169

[13] T. Minari, Y. Miyata, M. Terayama, T. Nemoto, T. Nishinaga, K. Komatsu, S. Isoda, Appl. Phys. Lett. 88 (2006) 083514

[14] M. D. Austin, S. Y. Chou, Appl. Phys. Lett. 81 (2002) 4431

[15] F. Ante, D. Kälblein, T. Zaki, U. Zschieschang, K. Takimiya, M. Ikeda, T. Sekitani, T. Someya, J. N. Burghartz, K. Kern, H. Klauk, Small 8 (2012) 73

[16] K. Tsukagoshi, F. Fujimori, T. Minari, T. Miyadera, T. Hamano, Y. Aoyagi, Appl. Phys. Lett. 91 (2007) 113508

[17] K. Nakayama, W. Ou-Yang, M. Uno, I. Osaka, K. Takimiya, and J. Takeya, submitted to Org. Electron.

[18] M. Kitamura, Y. Arakawa, Appl. Phys. Lett. 95 (2009) 023503.

[19] R. Nakahara, M. Uno, T. Uemura, K. Takimiya, J. Takeya, Adv. Mater. 24 (2012) 5212. 
[20] W. Ou-Yang, M. Weis, K. Lee, T. Manaka, M. Iwamoto, Jpn. J. Appl. Phys. 50 (2011) 04DK10.

[21] Y. Xu, R. Gwoziecki, I. Chartier, R. Coppard, F. Balestra, G. Ghibaudo, Appl. Phys. Lett., 97 (2010) 063302.

[22] P. V. Pesavento, R. J. Chesterfield, C. R. Newman, and C. D. Frisbie, J. Appl. Phys. 96 (2004) 7312.

[23] N. Koch, A. Kahn, J. Ghijsen, J. J. Pireaux, J. Schwartz, R. L. Johnson, and A. Elschner, Appl. Phys. Lett. 82 (2003) 70.

[24] T. Minari , T. Miyadera , K. Tsukagoshi , Y. Aoyagi , H. Ito , Appl. Phys. Lett. 91 (2007) 053508.

[25] F. Fujimori, K. Shigeto, T. Hamano, T. Minari, T. Miyadera, K. Tsukagoshi, Y. Aoyagi, Appl. Phys. Lett., 90 (2007) 193507

[26] S. P. Tiwari, W. J. Potscavage Jr., T. Sajoto, S. Barlow, S. R. Marder, B. Kippelen, Org. Electron. 11 (2010) 860

[27] U. Zschieschang, M. J. Kang, K. Takimiya, T. Sekitani, T. Someya, T. W. Canzler, A. Werner, J. Blochwitz-Nimoth, H. Klauk, J. Mater. Chem. 22 (2012) 4273.

[28] F. Dinelli, M. Murgia, P. Levy, M. Cavallini, F. Biscarini, Phys. Rev. Lett. 92 (2004) 116802

[29] P. V. Pesavento, K. P. Puntambekar, C. D. Frisbie, J. C. McKeen, P. P. Ruden, J. Appl. Phys. 99 (2006) 094504

[30] W. Ou-Yang, M. Weis, D. Taguchi, X. Chen, T. Manaka, M. Iwamoto, J. Appl. Phys. 107 (2010) 124506

[31] P. K. Koech, D. J. Gaspar et al., Chem. Mater., 22 (2010) 3926

[32] W. Ou-Yang, M. Weis, T. Manaka, M. Iwamoto, Jpn. J. Appl. Phys. 51 (2012) 024102

[33] W. Ou-Yang, X. Chen, M. Weis, T. Manaka, M. Iwamoto, Jpn. J. Appl. Phys. 49 (2010) 04DK04

[34] Y. D. Park, D. H. Kim, Y. Jang, J. H. Cho, M. Hwang, H. S. Lee, J. A. Lim, K. Cho, Org. Electron, 7 (2006) 514

[35] M.-H. Chen, J. Hou, Z. Hong, G. Yang, S. Sista, L.-M. Chen, Y. Yang, Adv. Mater. 21 (2009) 4238 


\section{List of Figure Captions}

Figure 1: Transfer characteristics in (a) linear regime (blue lines) and (b) saturated regime (blue lines), and (c) output characteristics of a typical TC/BG OFET with an inserted acceptor layer of $\mathrm{F}_{6}$ TNAP. Results of an $\mathrm{F}_{4} \mathrm{TCNQ}$ inserted OFET are also added (red lines) in (a) and (b) for direct comparison. The inset in (a) is a sketch of the OFET structure. The channel width is $500 \mu \mathrm{m}$ and channel length is $60 \mu \mathrm{m}$. The active layer of $C_{10}$-DNTT has a thickness of $15 \mathrm{~nm}$.

Figure 2: A typical result of contact resistance evaluation from linear regime for the OFET with an inserted acceptor layer of $F_{6}$ TNAP from the M-TLM method. The lines are linear fitting results and the thickness of $C_{10}$-DNTT active layer is $15 \mathrm{~nm}$.

Figure 3: (a) Gate voltage dependent contact resistance and (b) channel length

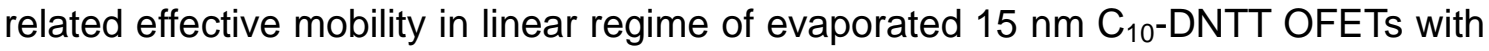
an acceptor layer of $\mathrm{F}_{6} \mathrm{TNAP}$ or $\mathrm{F}_{4} \mathrm{TCNQ}$. (c) Gate voltage dependent contact resistance of $\mathrm{F}_{6}$ TNAP layer incorporated $\mathrm{C}_{10}$-DNTT OFETs with different active layer thicknesses. The inset is a sketch of equivalent circuit of total drain-source resistance. Experimental error bars are included.

Figure 4: (a) Gate voltage dependent contact resistance and (b) channel length influenced contact resistance ratio (contact resistance over total resistance at gate

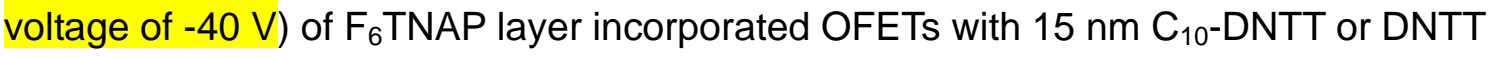
as the active layer. 
Figures

Figure 1a

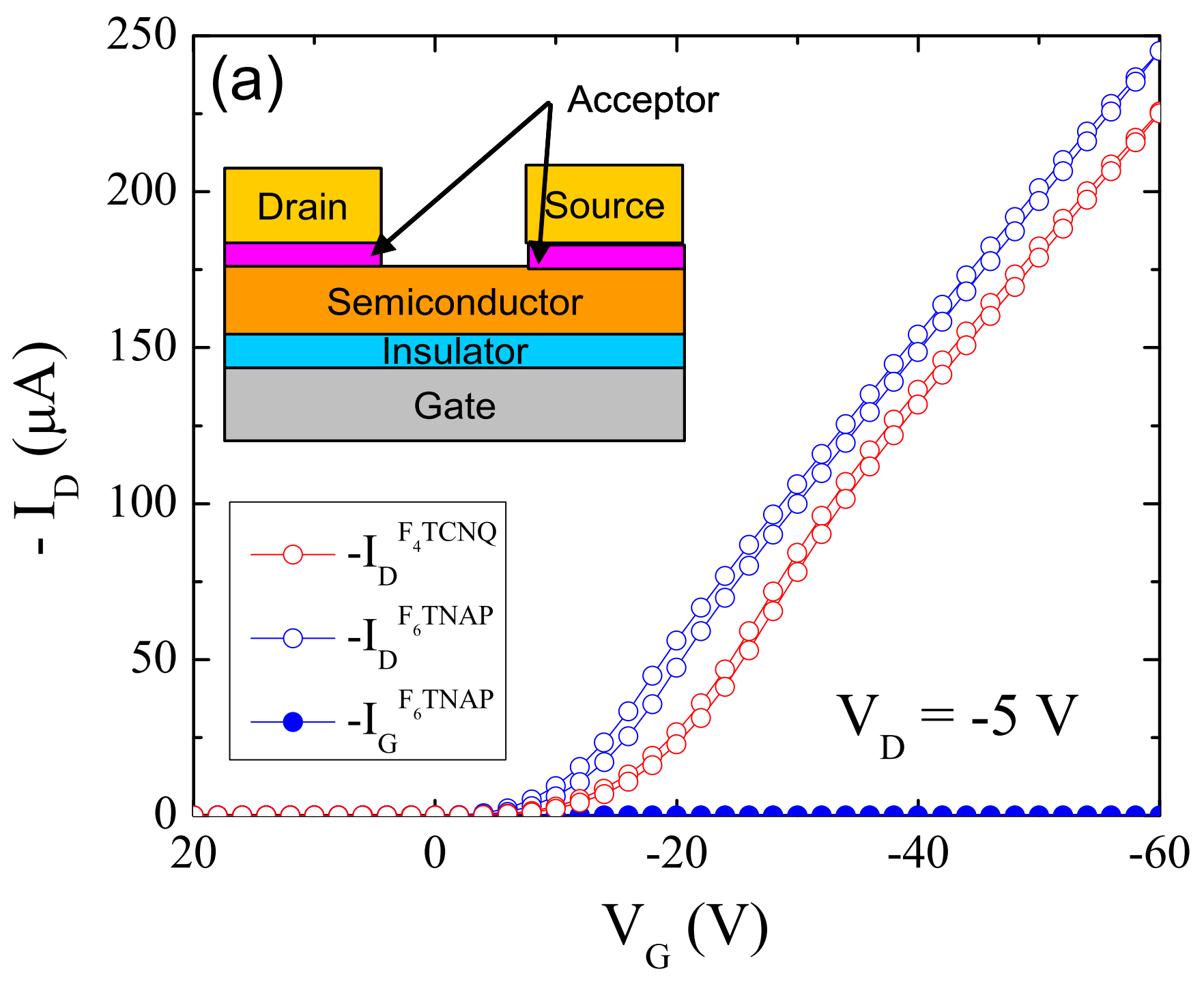


Figure 1b

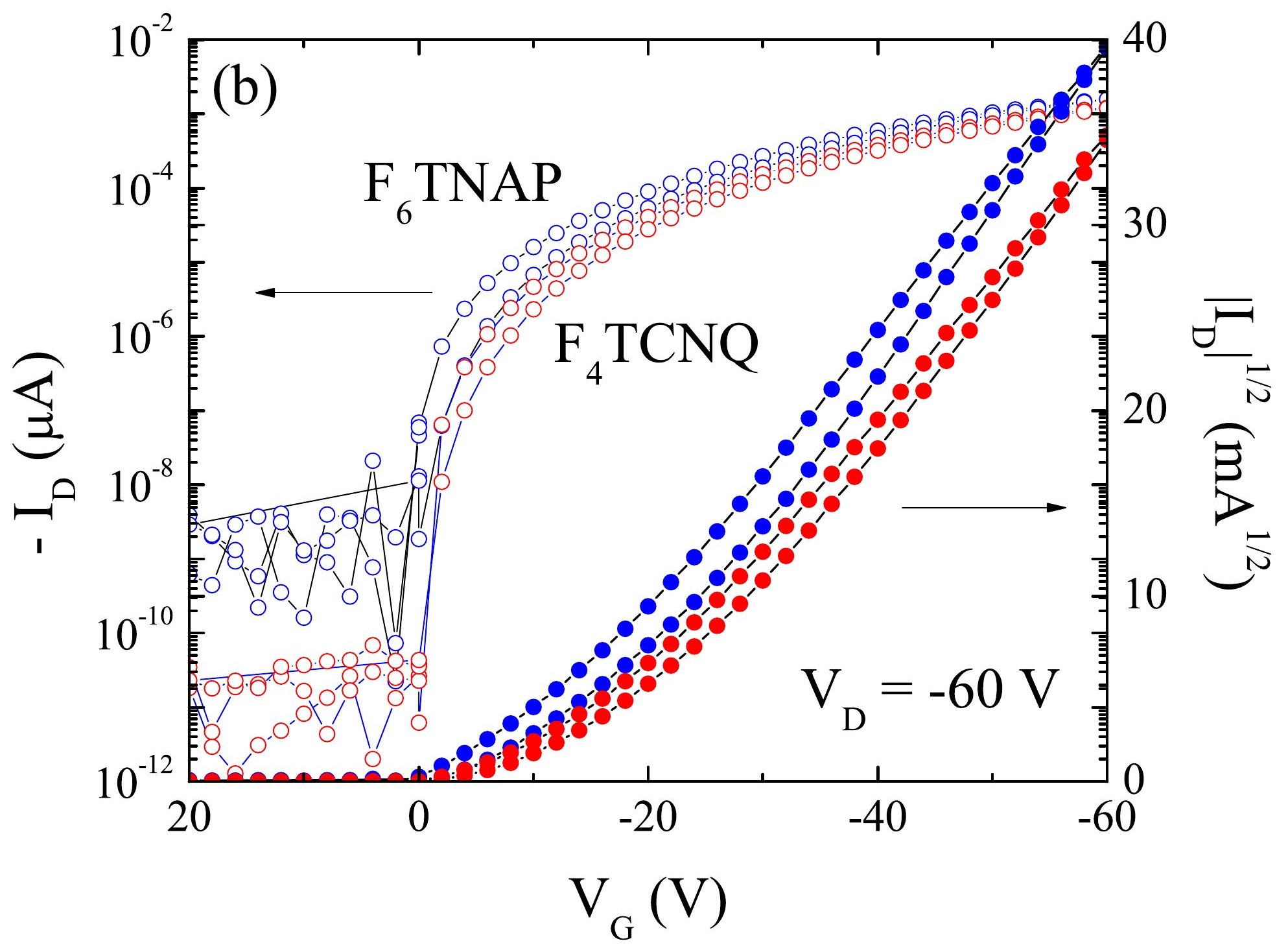




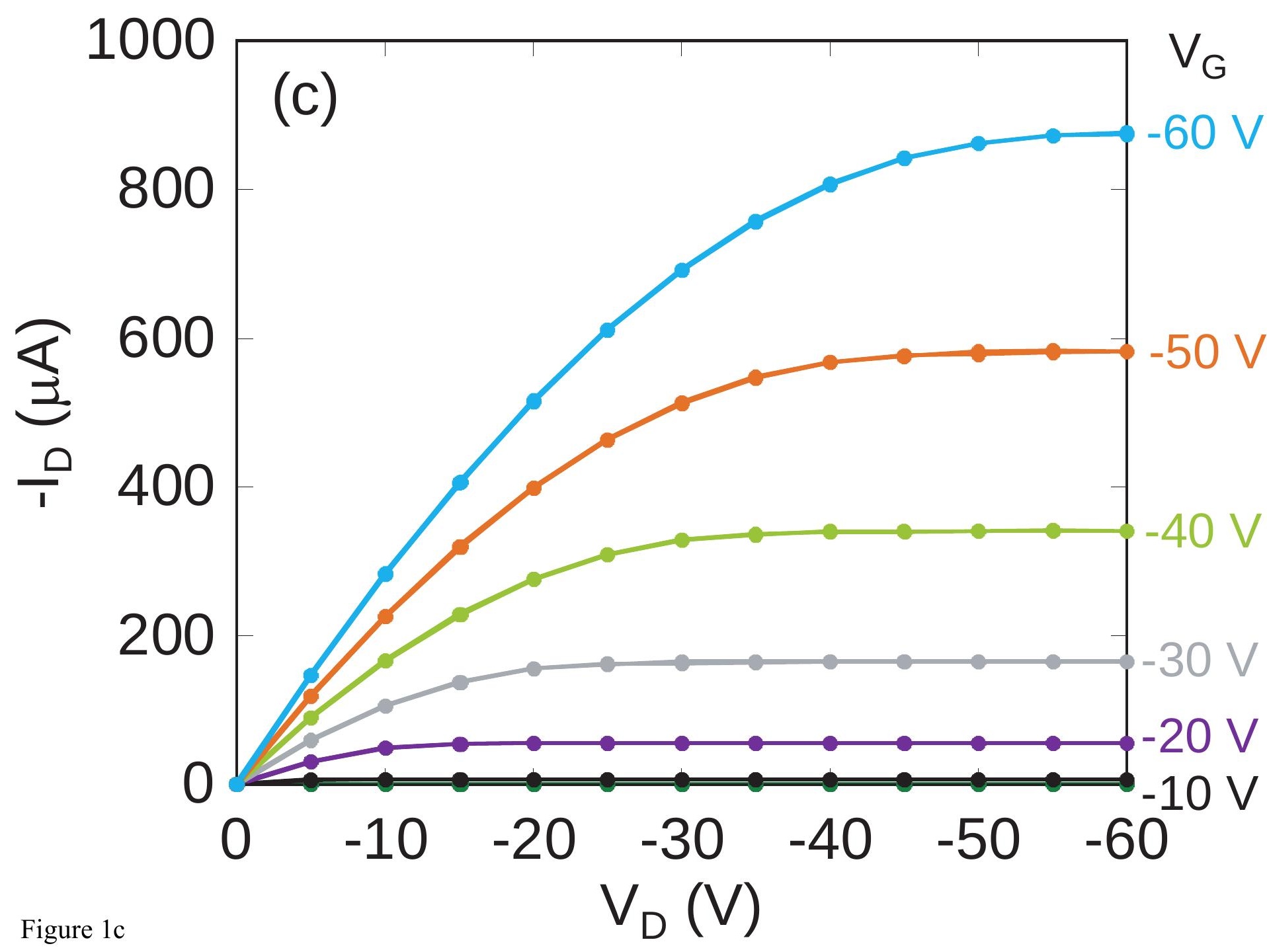




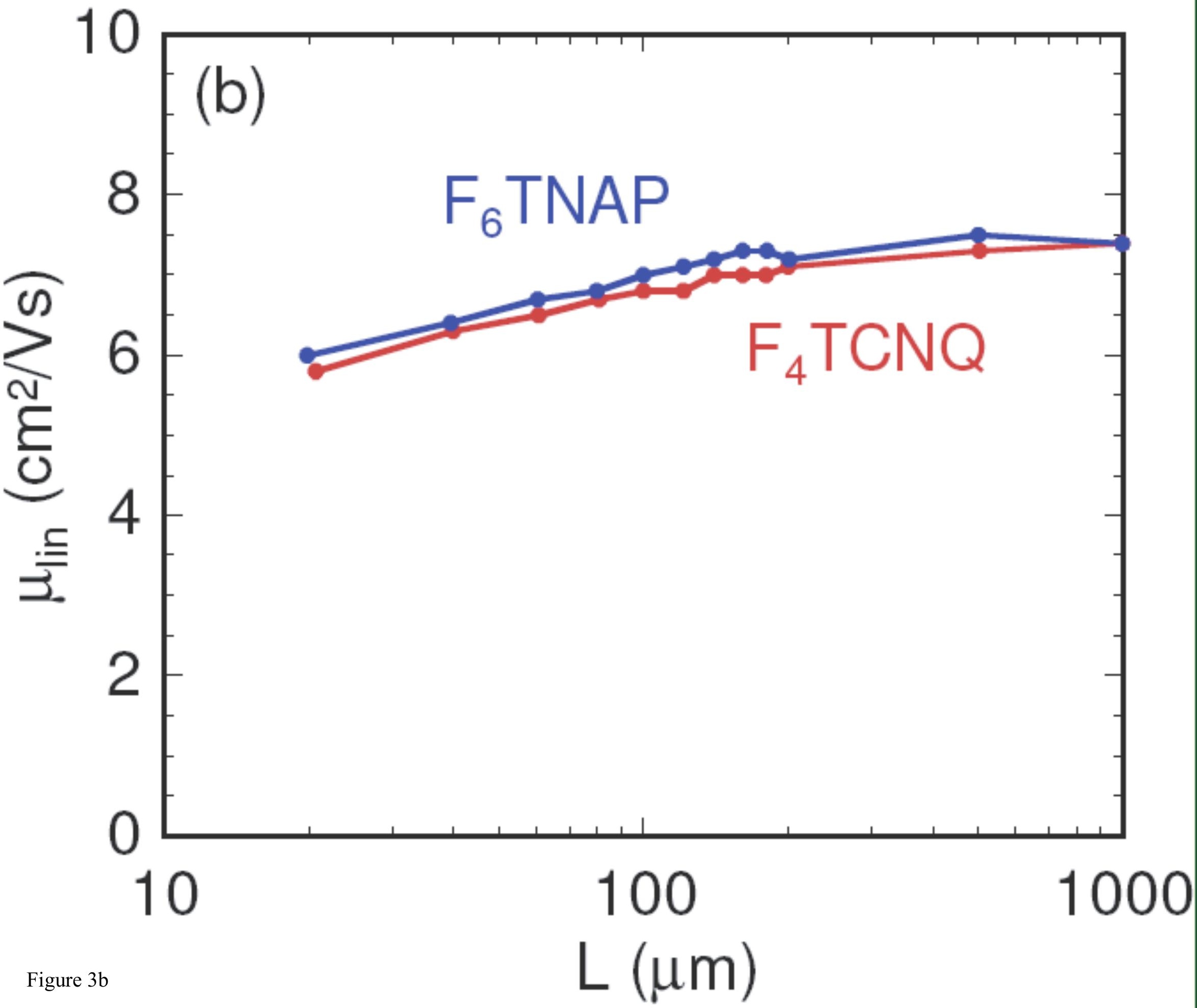




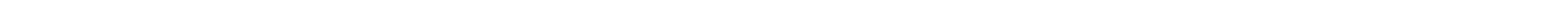




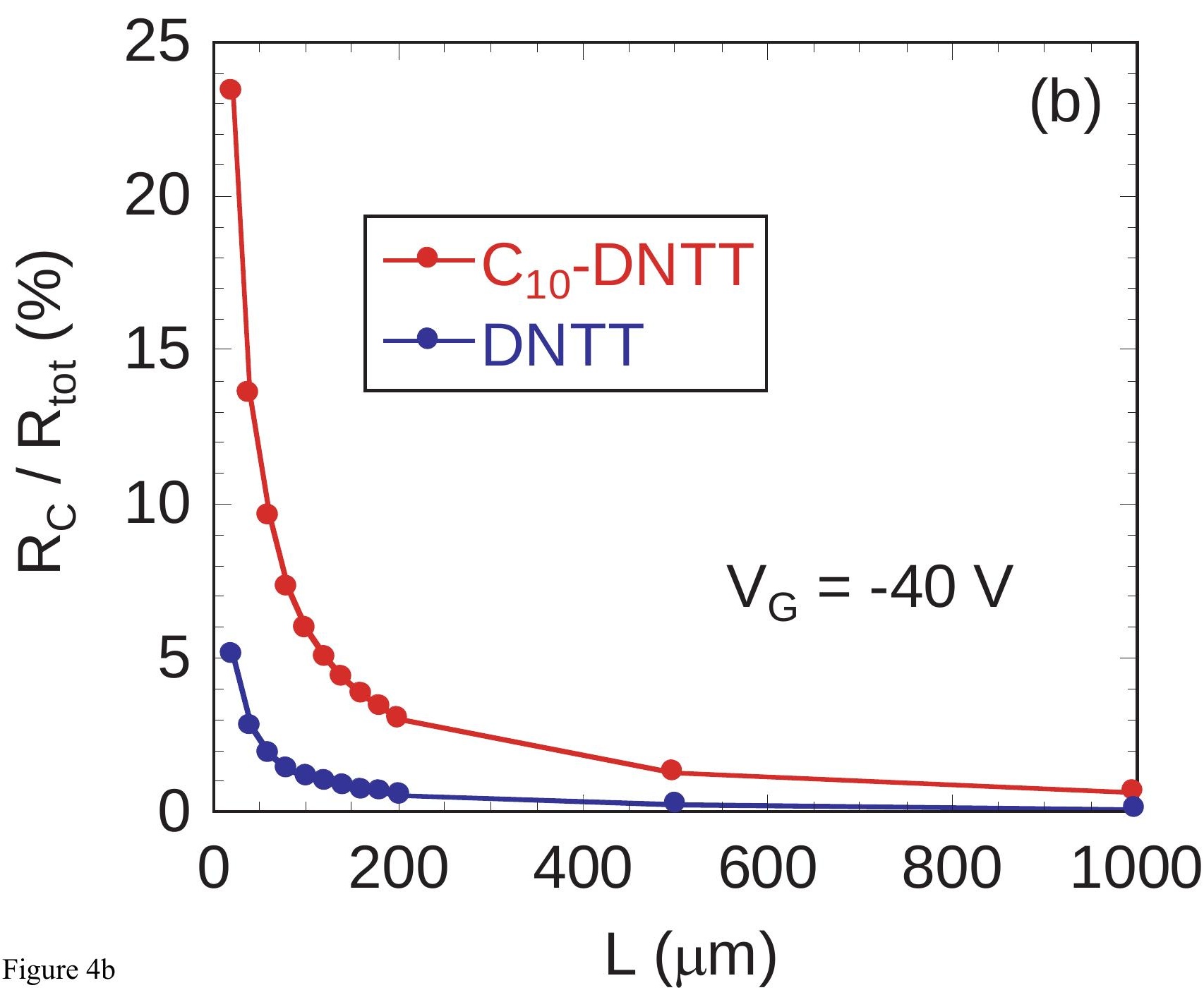




\section{Graphical abstract}
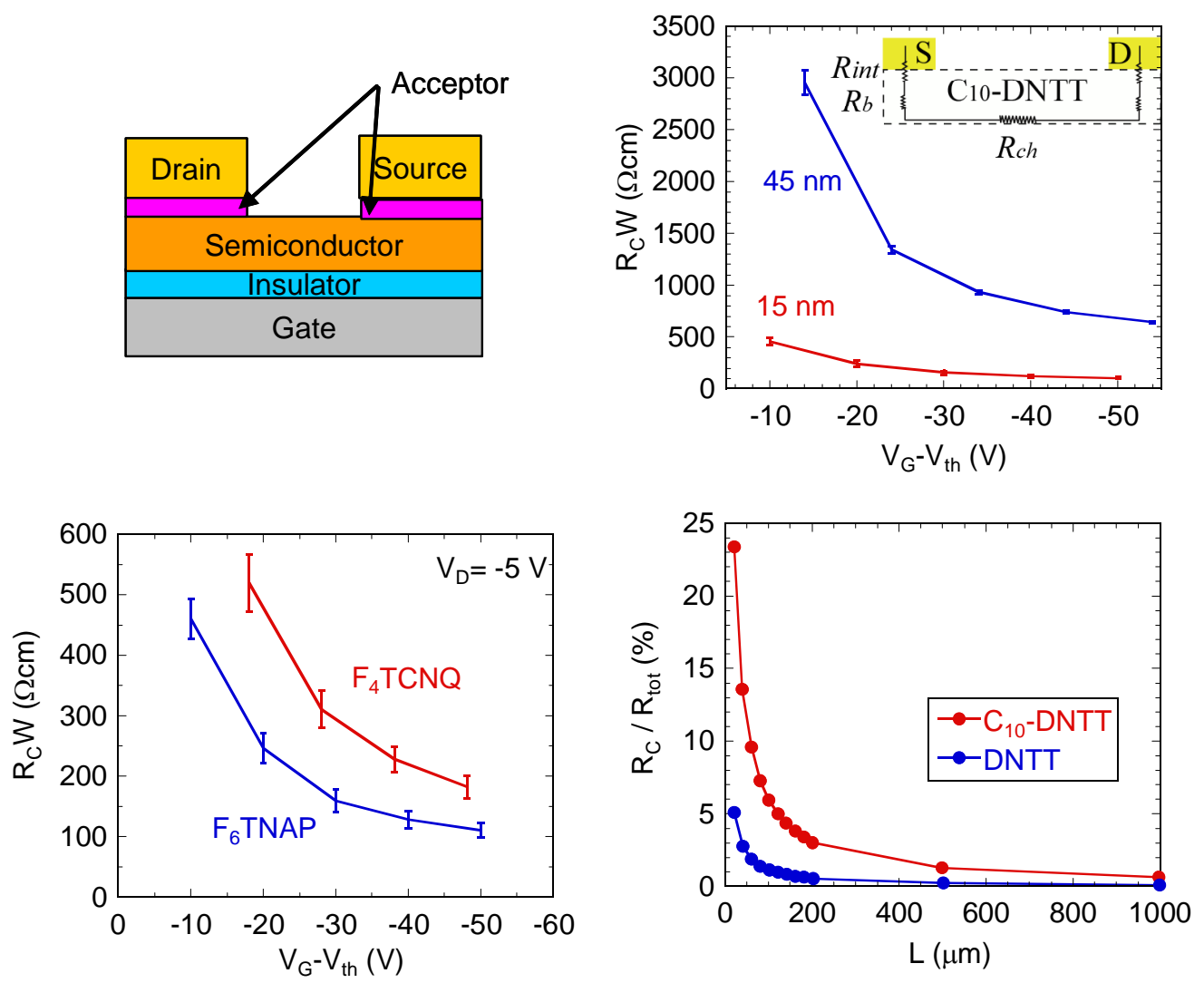